\title{
Training the trainable cells of the immune system and beyond
}

\author{
The fourth Innate Immune Memory meeting was held in the historic city of Nijmegen, in the eastern-central part \\ of the Netherlands, to discuss the basic and translational aspects of innate immune memory, popularly known as \\ 'trained immunity'.
}

$\mathrm{H}$ ost immune responses are classically divided into two parts: an innate immune response, poised to act rapidly and non-specifically after an invading pathogen or toxin is encountered, and an adaptive immune response, composed of a small number of cells that act with high specificity and build up immunological memory. However, in recent years, the dogma that only adaptive immunity can generate immunological memory has been challenged by studies showing that innate immune responses in plants and invertebrates (organisms lacking canonical adaptive immune responses) can mount resistance to reinfection ${ }^{1}$. In addition, several studies have demonstrated that, in mouse and human cells, priming with Candida albicans or the fungal cell-wall component $\beta$-glucan non-specifically induces enhanced secondary responses. Furthermore, in certain mammalian models of vaccination, protection from reinfection has been shown to occur independently of $\mathrm{T}$ and B lymphocytes. These observations led to a new concept in immunology termed 'innate immune memory', or 'trained immunity' (Fig. 1). Innate immune memory differs from adaptive memory in many aspects, including the lack of gene rearrangements, the involvement of epigenetic reprogramming, the type of cells involved (innate cells versus $\mathrm{T}$ and $\mathrm{B}$ lymphocytes), and the receptors engaged in pathogen or antigen recognition (selective pattern-recognition receptors versus antigen-specific T cell and B cell receptors) ${ }^{1}$. The goal of the meeting, divided into seven sessions over 2 days, was to bring leading scientists, $\mathrm{PhD}$ students and postdoctoral researchers to present their new findings and exchange ideas with colleagues. It was a well-organized meeting, designed in such a way as to allow at least one young investigator to present with the established investigators and to provide sufficient time for in-depth discussions on the different subjects covered at the meeting.

\section{Evolutionary aspects}

Jos van der Meer (Radboud University Medical Center (Radboudumc)) chaired the first scientific session, and Joachim L. Schultze (University of Bonn) kicked

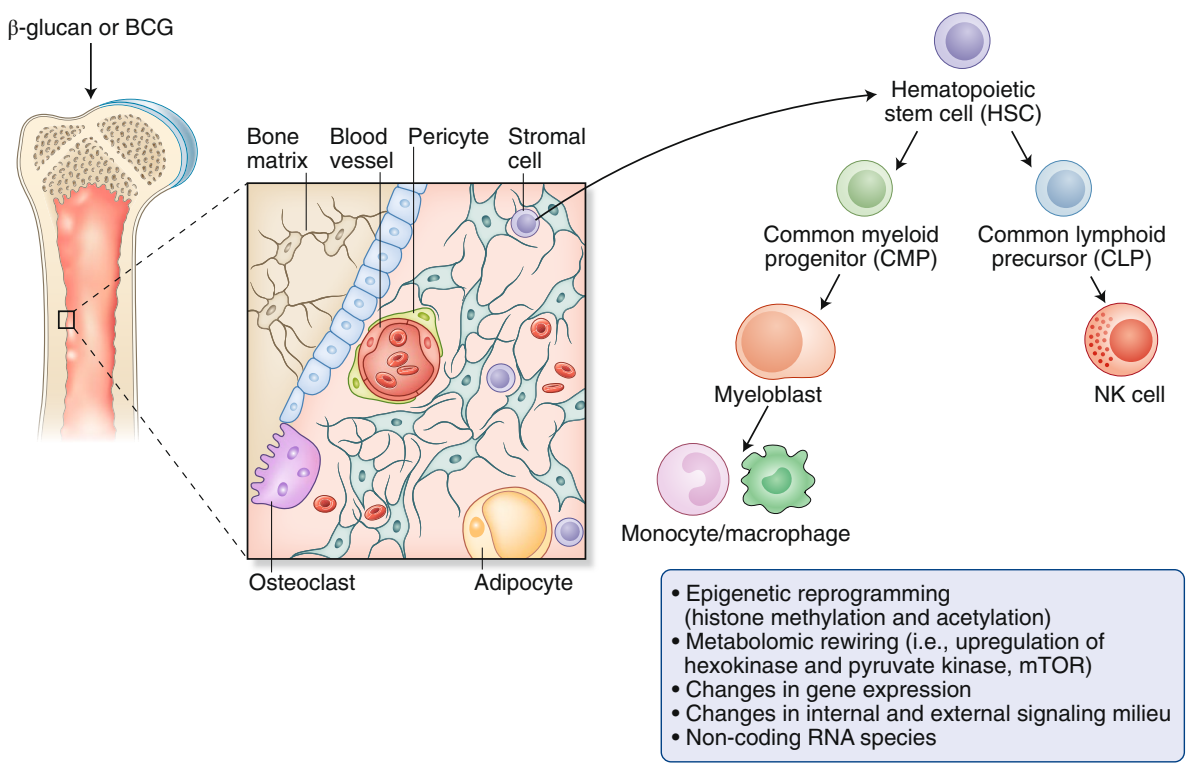

Fig. 1 | Schematic representation of the concept of 'training immunity'. BCG or $\beta$-glucan induces reprogramming of HSCs and multipotent progenitors through epigenetic reprogramming, metabolomic rewiring and changes in gene expression signatures, along with changes in both the internal and external signaling milieu. This training inherited by myeloid cells, that is, monocytes, macrophages and NK cells, allows them to respond more robustly to microbial or non-microbial stimulation.

Credit: Debbie Maizels/Springer Nature

off the meeting by summarizing the evolutionary continuum of innate and adaptive immune memory in the host's response to pathogens. The species we encounter today are present because of their adaptive evolution, that is, their ability to rapidly and reversibly modify their properties to maximize fitness in a changing environment. Immunological memory is considered one of the many principal components of adaptive evolution that has helped the host to survive upon reinfection. Therefore, immune memory is a general characteristic of host defense in all living organisms beyond vertebrates (including invertebrates and plants, but also bacteria and archaea). More importantly, immunological memory is much more widespread and diverse than previously suggested. As many as six varieties of immune memory have been described: classical adaptive memory in vertebrates, natural killer (NK) cells' immune memory, trained immunity in myeloid cells, priming in invertebrates, immunological memory in plants (for example, systemic acquired resistance, SAR), and CRISPR-Cas9-based memory in bacteria and archaea ${ }^{2}$. The evolution of immune memory in various groups of organisms is a continuum that started with the development of epigenetic mechanisms responsible for increasing the magnitude and speed of the immune response upon reinfection and continued thereafter with the buildup of specificity in vertebrates by mechanisms including gene recombination and clonal selection. Whereas magnitude and kinetics amplification by epigenetic rewiring characterize a more primitive form of innate immune memory, higher magnitude, kinetics and specificity characterize the refined adaptive immune memory in vertebrates ${ }^{2}$. At the end of his presentation, Schultze highlighted a few questions that remain to be addressed. For example, 


\section{Box 1 | Future directions}

The open-ended questions yet to be addressed are as follows:

- Monocytes are known be short-lived ( 1-7 days). How is memory information stored, and does this allow monocytes to survive longer?

- Is there a specific memory monocyte subset yet to be identified?

- What are the roles of hematopoiesis and bone marrow in trained immunity?

- Is chromatin-based-immune training applicable to all the myeloid cells?

- What is the role of the adaptive immune system in the maintenance of innate memory?
- What are the dynamics of innate memory? What is the division of labor between adaptive and innate memory?

- Do vaccines play a role? Which vaccines induce trained immunity, and what is the underlying mechanism?

- How can we control vaccine-induced innate memory?

- Can existing adjuvants be exploited for training and, if not, can novel adjuvants be developed for this purpose? Can this framework be used to build an antigen-nonspecific vaccine to protect individuals at risk or during disease outbreaks? how can general rules be established to distinguish between memory and adaptation? Because innate immunological memory has multiple dimensions, what are the different molecular mechanisms that determine the strength, duration (shortterm versus long-term memory), speed, specificity (to the target) and extinction (via downregulation mechanisms)?

Joseph C. Sun (Memorial Sloan Kettering Cancer Center) further highlighted that innate memory is not a phenomenon restricted to monocytes and macrophages. NK cells also show avidity selection (specific memory features) during human cytomegalovirus (HCMV) infection. Antigen-specific NK cell memory has been demonstrated for a wide variety of antigens, including haptens (virus-like particles), and in the context of infections such as influenza, vaccinia and others ${ }^{3,4}$. Almost a decade ago, Paust et al. showed that administration of inactivated vesicular stomatitis virus induces an NK cell population that confers specific protection to lethal viral challenge in T celland B cell-deficient mice ${ }^{5}$. Using assays such as assay for transposase-accessible chromatin using sequencing (ATAC-seq) and transcriptional profiling (RNA-seq), Sun's group investigated how chromatin modifications dictate transcriptional fates that produce the naive, effector and memory states during mouse CMV infection. They discovered that NK cells and $\mathrm{CD}^{+} \mathrm{T}$ cells possess shared epigenetic and transcriptional programs that underlie host immunological memory. The striking similarity between $\mathrm{NK}$ cell and $\mathrm{CD}^{+} \mathrm{T}$ cell methylation patterns in $\mathrm{HCMV}^{+}$individuals was also previously documented ${ }^{6}$. NK cells can develop specific and non-specific memory resembling a trained immunity process. Though chromatin remodeling seems to be at the heart of NK cell memory, changes in receptors and their induction cannot be ruled out.

Godfrey Temba (Radboudumc) shared his research on the role of non-genetic host and environmental factors (diet change and urbanization) on the human immune response. In a comparison study, he compared the levels of circulating plasma cytokines (that is, interleukin (IL)-6, IL-1 $\beta$, IL-1 receptor antagonist (IL-1Ra), IL-18 and IL-18-binding protein (IL-18BPa)) and circulating adipokines, along with the genomes, transcriptomes and metabolomes of subjects living in rural versus urban areas in Tanzania. Not surprisingly, the urban and rural subpopulations showed distinct patterns, and greater inflammation was observed in the urban participants. This research suggests that environmental changes define immunological footprints. However, further investigations are required to identify the roles of other factors, including vaccine records, exposure to other infectious diseases and hygiene.

Anna C. Aschenbrenner (University of Bonn) delivered a presentation on the long-term adaptation of fatty-acid-primed monocytes. Though the intertwined relationship between immune cell energy metabolism and function has been reported on since the early 1960s, recent technological advancements as described in, for example, transcriptomic, epigenetic, functional and fate-mapping studies have helped to uncover the fundamental role of energy metabolism in immune cell function. In considering the role of myeloid cells in innate immune responses, an increasing amount of attention has been devoted to the characterization of energy metabolism. Aschenbrenner's group treated human monocytes with the fatty acids palmitic acid and oleic acid and observed different immune responses. Though priming of monocytes with palmitic acid and oleic acid resulted in reprogramming, the secondary response did not show the typical 'trained immunity' response. The authors believe that this finding occurred because the monocytes undergo adaptation-a model recently explained in great detail ${ }^{7}$. Furthermore, the changes in chromatin structure (determined with ATAC-seq) also showed no major driver of reprogramming. Their research is now focused on assessing the heterogeneity of the response (distinguishing responsive versus nonresponsive cells) to compare the response to $\beta$-glucan and the effects on methylation and histone modification during primary and secondary responses.

\section{Cellular substrates of innate memory}

This session was chaired by Niels Riksen (Radboudumc), and the first speaker of the session was Keiko Ozato (US National Institutes of Health). She shared her research on the role of interferons in inducing epigenetic memory. Interferon (IFN)- $\beta$ stimulation generates transcriptional memory in fibroblasts, conferring faster and greater gene expression after restimulation. The memory can be inherited through multiple cell divisions and can induce improved antiviral protection. This mechanistic analysis suggests that IFN memory is not due to enhanced IFN signaling or retention of transcription factors on the IFN-stimulated genes but instead is due to acquisition of the histone variant histone $\mathrm{H} 3.3$ and trimethylated histone $\mathrm{H} 3$ Lys 36 chromatin marks on memory IFN-stimulated genes. Similar memory can also be observed in bone marrow macrophages after IFN- $\gamma$ stimulation. This finding suggests that IFNs can modify the shape of the innate immune response . $^{8}$

Stephanie Fanucci (University of Cape Town) shared her research on the roles of long non-coding RNAs (lncRNAs) in trained immunity. In the past decade, these non-protein-encoding transcripts have been recognized as key regulators of gene expression. Their roles in physiological processes and adverse pathophysiological disorders have been widely reported. Trimethylated H3 Lys 4 (H3K4me3) is an epigenetic modification to the DNA packaging protein histone $\mathrm{H} 3$ and is often involved in the regulation of gene expression?. In recent years, many groups have reported that trained immunity is caused by epigenetic reprogramming at the level of histone methylation and acetylation. The trained cells, after a brief exposure to $\beta$-glucan, the Bacillus Calmette-Guérin (BCG) vaccine or oxidized-low-density lipoprotein, are characterized by an enrichment of the 
activating histone modifications $\mathrm{H} 3 \mathrm{~K} 4 \mathrm{me} 3$ and monomethylated $\mathrm{H} 3$ Lys 4, and the trained phenotype can be prevented by coadministration of pharmacological inhibitors of histone methyltransferases. Fanucci highlighted the contributions of lncRNAs to H3K4me3 promoter priming in trained immunity. She discussed the interactions between certain lncRNAs and $\mathrm{H} 3 \mathrm{~K} 4 \mathrm{me} 3$ modifications and the influence that this might have on promoter priming in trained immunity.

Florian Wimmers (Stanford University) presented his findings on changes that occur in the chromatin landscape after inoculation of human volunteers with inactivated vaccines. Using a variety of stimuli up to 180 days postvaccination, Wimmers and colleagues analyzed the changes in global histone modifications of circulating myeloid dendritic cells, plasmacytoid dendritic cells and monocyte subsets (classical monocytes and non-classical monocytes) by using a powerful tool called epigenetic landscape profiling using cytometry by time-of-flight (EpiTOF). There was substantial epigenetic reprogramming of monocytes in comparison to naive cells, thus suggesting the involvement of trained immunity in human vaccination.

\section{Innate memory mechanisms}

The post-lunch session was on innate immune memory mechanisms and was chaired by Giuseppe Matarese. The first speaker, Triantafyllos Chavakis (Technische Universität Dresden), discussed the effects of $\beta$-glucan training on granulopoiesis. His findings suggest that trained immunity induces a myelopoiesis bias through metabolic rewiring of hematopoietic progenitors $^{10}$. His group has taken one step further and investigated whether training of the innate immune response by using $\beta$-glucan can inhibit tumor growth. The authors observed inhibition in two models, B16-F10 melanoma and Lewis lung carcinoma. The use of $\beta$-glucan as an immune adjuvant in the treatment of solid and hematological malignancies is not new. However, the antitumor activity of $\beta$-glucan was previously proposed to relate to signaling through the receptor dectin- $1^{11}$. The new findings - namely, training immunity by using $\beta$-glucan to reverse the hijacking of granulopoiesis by tumors-are exciting and will be of interest to many.

Maziar Divangahi (McGill University Health Centre) further highlighted that hematopoietic stem cells (HSCs) can be educated to imprint mononuclear phagocytes to maintain their memorylike protective capacity against a virulent bacterial pathogen such as Mycobacterium tuberculosis. The research suggests that after exposure to BCG (or $\beta$-glucan), the transcriptional landscape of HSCs and multipotent progenitors leads to enhanced myelopoiesis. Furthermore, this epigenetic memory is transmitted to mature myeloid cells, thus leading to the generation of trained monocytes and macrophages. BCG-educated HSCs generate epigenetically modified macrophages that provide significantly better protection against virulent $M$. tuberculosis infection than naive macrophages ${ }^{12}$.

Carlos del Fresno (Centre d'Immunologie de Marseille-Luminy) provided a presentation on how trained immunity can be boosted by targeting the $\mathrm{SH} 2$-domaincontaining inositol $5^{\prime}$ phosphatase SHIP1, a negative regulator of $\beta$-glucan-induced training. Using the pharmacological inhibitor 3AC, they demonstrated enhanced proinflammatory-cytokine production and better protection against Candida infection in both mouse and human peripheral blood mononuclear cells (PBMCs). The results suggest that pharmacological agents such as $3 \mathrm{AC}$ can be considered as potential therapeutic approaches to boost trained immunity ${ }^{13}$.

Bérengère de Laval (French Institute of Health and Medical Research) discussed the recent findings on the contribution of CCAAT/enhancer binding protein $\beta(\mathrm{C} / \mathrm{EBP} \beta)$ to the myeloid signature of hematopoietic stem cells. C/EBP $\beta$ is well known to cooperate with the switch/sucrose non-fermentable (SWI/SNF) nucleosomeremodeling complex to induce or repress the expression of target genes and ultimately regulate the proliferation, differentiation, metabolism and survival of many different cell types including HSCs. These findings suggest that epigenetic priming in HSCs and the changes transmitted from HSCs and progenitors to the mature myeloid cells are important, because they improve understanding of the roles of HSCs in trained immunity.

The second session on innate immune memory mechanisms was chaired by Zoltan Fehervari (Nature Research). Robin Choudhury (University of Oxford) presented data on hyperglycemia-mediated induction of trained immunity. His research suggests that hyperglycemia alters chromatin accessibility and implicates transcription factors such as RUNX1 and PU.1.

Interestingly, human atherosclerotic plaque macrophages showed differential expression of RUNX1 and PU.1, and inhibition of RUNX1 removed the trained-immunity phenotype in vitro. This type of research is forcing the field to consider identifying new strategies for developing diagnostic assays for patients with impaired glucose tolerance.
Willem Mulder (Icahn School of Medicine at Mount Sinai) presented how nanobiologics can be designed to target trained immunity. He shared data on how targeting the underlying mechanisms of trained immunity, such as metabolic and epigenetic pathways, can provide a compelling framework for developing new treatment methods for autoimmune disorders, chronic inflammatory conditions, cardiovascular diseases and allergies ${ }^{14}$.

Kenneth Walsh (Boston University School of Medicine) delivered the closing keynote of day one on clonal hematopoiesis, a condition in which a substantial proportion of mature blood cells are derived from a single clone. Recently, several investigators have demonstrated that innate immune cells can inherit memory from their progenitors; thus, the bone marrow is an integral component of trained immunity ${ }^{12}$. Identifying the clones (in bone marrow) and the distinct factors governing their generation may enable trained immunity to be harnessed in the clinic.

\section{Epigenetics}

Leo Joosten chaired the first scientific session on day two, and Luis Barreiro (University of Chicago) started the conversation on inter- and intra-individual variation in the innate immune response. The immune system is composed of different cell lineages that reside in primary and secondary lymphoid organs and tissues throughout the body, as well those that transit through the peripheral blood and lymphatic systems. Moreover, variations depend on cell type, state and location. In recent years, technological advances in single-cell RNA-seq and advanced computational methods have revolutionized understanding of immunology. Using RNA-seq, Barreiro and colleagues identified the genes responsible for variation in trained immunity, particularly in BCG training, which can be exploited for therapeutic applications.

Ramnik Xavier (Harvard University) extended the discussion and shared his data on trained immunity. Subsequently, Michel Vierboom (Biomedical Primate Research Centre) presented research on non-human primates, showing the increased production of cytokines linked to trained immunity (TNF, IL-6 and IL-1 $\beta$ ) 2 weeks after intravenous BCG vaccination. Moreover, the work from Vierboom and colleagues demonstrates that mucosal vaccination with either MTBVAC (a genetically attenuated M. tuberculosis-derived TB vaccine candidate) or BCG is superior to standard intradermal vaccination in the induction of trained immunity. For mucosal BCG 
vaccination, they have recently shown improved protection and prevention of infection relative to standard intradermal delivery. They are now investigating whether mucosal vaccination also leads to metabolic rewiring and genome-wide epigenetic reprogramming. Given the literature on trained immunity, genomewide epigenetic reprogramming in innate immune cells is expected. Previous work from the same group has already demonstrated an association between innate immune responses and reduced TB disease severity in the non-human primate species. In a publication by Dijkman et al., they have shown that disease-susceptible rhesus macaques (Macaca mulatta) present a skewed anti-inflammatory profile of peripheral monocytes, while diseaseresistant cynomolgus macaques (Macaca fascicularis) display a more prominent local proinflammatory innate cytokine release profile (a well-known manifestation of trained immunity $)^{15}$.

Spencer Gill (Department of Surgery, East Tennessee State University) shared his findings on how trained immunity can be targeted to reverse the age-related changes in the fecal microbiome. Age-related perturbations in the gut microbiome and the underlying mechanism that leads to various age-associated pathological states is being intensively studied. The proposed mechanism includes direct modulation of the gut microbiome (to stabilize immunosenescence), augmentation of antioxidant activity, regulation of host-fat deposition and metabolism, suppression of insulin resistance, improvements in mucosal barrier integrity and immune homeostasis, elevated production of shortchain fatty acids, reducing the expression of gut peptides involved in lipid metabolism and glucose homeostasis, and upregulation of particular genes involved in xenobiotic metabolism to improve or restore gut eubiosis. However, the approach followed by his group - targeting trained immunity-is certainly a new direction that warrants further investigation.

\section{Trained immunity in disease}

The next session on disease pathophysiology, chaired by Leo Joosten and Niels Riksen (Radboudumc), gave a comprehensive overview of trained immunity and atherosclerosis, emphasizing the role of the bone marrow in understanding this disease. In recent years, evidence has accumulated suggesting that many cardiovascular risk factors, both traditional and non-traditional (for example, nutrients and microbiota), induce epigenetic reprogramming of innate immune cells, a well-defined factor of trained immunity. Therefore, use of pharmacological agents to influence epigenetic remodeling or trained immunity should be considered a promising tool for the future treatment of atherosclerosis.

Shabaana Khader (Washington University School of Medicine) shared her recent findings on unique single-nucleotide polymorphisms in the RNA polymerase subunit $\beta$ gene $(r p o B)$ of multidrugresistant $M$. tuberculosis strains that can alter macrophage metabolism ${ }^{16}$. This is another perspective to consider when manipulating metabolism rewiring and epigenetic reprogramming-well-known characteristics of 'trained immunity'.

Laura Conejero (Inmunotek, Alcalá de Henares) shared her experience with MV130, a new whole heat-inactivated polybacterial preparation (PBP) vaccine, which is being developed by Inmunotek SL, for the treatment of bronchospasm, non-cystic fibrosis. Daily sublingual administration of MV130 significantly decreased the rate of respiratory infections in patients with recurrent respiratory tract infections. Further investigation indicated that MV130 triggered Toll-like receptor (TLR) and nucleotide-binding and oligomerization domain (NOD)-like receptor (NLR) signaling pathways on dendritic cells, stimulating the release of the cytokines TNF, IL- 6 and IL-1 $\beta$-hallmarks of trained immunity ${ }^{17}$. Kyle Cunningham (Trinity College Dublin) shared data suggesting that innate immune cells can be trained to be more anti-inflammatory after exposure to products of a helminth pathogen. More importantly, this training protects mice against the induction of a $\mathrm{T}$ cell-mediated autoimmune disease ${ }^{18}$.

\section{Prophylaxis and treatment}

The final session of the conference focused on prophylaxis and treatment of trained immunity. Reinout van Crevel (Radboudumc) chaired the session, and Elisa Jentho (University Hospital Jena) presented on the protective role of hemeinduced trained immunity against sepsis. The involvement of inflammatory responses in sepsis is well documented. A recent study has demonstrated that sepsis induces a long-lasting state of trained immunity in bone marrow monocytes ${ }^{19}$. Jentho's research suggests that heme, a well-known activator of innate immune cells, such as macrophages and neutrophils, can induce trained immunity. This interesting finding might shed new light on the complex aftermath of sepsis and open up a new pathophysiological framework in need of further research. Christine Stabell-Benn (University of Southern Denmark) and Nigel Curtis
(University of Melbourne) highlighted the non-specific effects of vaccines. Both drew attention to how vaccine-development strategies should be rethought. Trainedimmunity-based vaccines, which can stimulate both non-specific and specific immune responses, might provide a broader protection far beyond the conventional vaccines currently used.

The final keynote was delivered by Michael Skinner (Washington State University), who has pioneered the understanding of epigenetic transgenerational inheritance-the transmission of epigenetic information through the germline. He led us all to think about the importance of the epigenome, namely gene regulatory feedback loops and chromatin modifications (DNA methylation and histone modifications), as well as longlived non-coding RNA molecules and their dominant roles in gene expression. In addition, human adaptations to the evolution process, that is, teaching and learning, culture (which influences learning), social behavior and so on, certainly play a role. After his presentation, Mihai G. Netea and Leo Joosten distributed the prizes for poster and oral presentations to the winners (junior investigators selected by a committee of senior investigators).

In summary, trained immunity is exhibited by all organisms studied to date. Though the underlying mechanisms are not fully understood, some principles have begun to emerge (Fig. 1). After exposure to BCG or $\beta$-glucan, reprogramming of HSCs and multipotent progenitors (and possibly niche cells) is altered through various mechanisms, including epigenetic reprogramming (histone methylation and acetylation), metabolomic rewiring (of hexokinase and pyruvate kinase, mTOR and so on), changes in gene expression signatures (mediated by microRNA and long-noncoding RNA expression), and changes in both the internal and external signaling milieu. This training is imposed on progenitors of monocytes, macrophages and NK cells, and transmitted to their progeny, thus allowing them to respond optimally after subsequent microbial or non-microbial stimulation. However, many open-ended questions are likely to occupy scientists over the next decade (Box 1). Research on trained immunity is still in its infancy, and continued studies in this burgeoning area will offer new therapeutic opportunities that can be personalized in the future.

\section{Concluding remarks}

This was a great meeting that brought together colleagues with different approaches to studying trained immunity. 
Several presenters shared new ideas, and all realized the collaborative efforts needed to know more about trained immunity. All attendees were enthusiastic about reconvening for the fifth edition of this conference, which will be held in Canada in 2020 (11-13 November). Thereafter, the sixth edition of the Innate Immune Memory conference will be held in 2021, in Naples, Italy. We are looking forward to it!

Upendra K. Kar (ID) $1 \times$ and Leo A. B. Joosten (D) ${ }^{1}$ Pittsburgh Trauma Research Center, Department of Surgery, University of Pittsburgh, Pittsburgh, Pennsylvania, USA. ${ }^{2}$ Department of Internal
Medicine and Radboud Centre for Infectious Diseases (RCI), Radboud University Medical Center, Nijmegen, the Netherlands.

$凶_{\boldsymbol{e} \text {-mail: karuk@upmc.edu }}$

Published online: 10 January 2020

https://doi.org/10.1038/s41590-019-0583-y

\section{References}

Netea, M. G. et al. Science 352, aaf1098 (2016).

2. Netea, M. G., Schlitzer, A., Placek, K., Joosten, L. A. B. \& Schultze, J. L. Cell Host Microbe 25, 13-26 (2019).

3. Paust, S., Blish, C. A. \& Reeves, R. K. J. Virol. 91 , e00169-17 (2017)

4. van den Boorn, J. G. et al. Immunity 44, 1406-1421 (2016).

5. Paust, S. et al. Nat. Immunol. 11, 1127-1135 (2010).

6. Schlums, H. et al. Immunity 42, 443-456 (2015).
7. Yuan, R., Geng, S. \& Li, L. Front. Immunol. 7, 497 (2016).

8. Kamada, R. et al. Proc. Natl Acad Sci. USA $\mathbf{1 1 5}$

E9162-E9171 (2018).

9. Fanucchi, S. \& Mhlanga, M. M. Front. Cell Dev. Biol. 7, 2 (2019)

10. Mitroulis, I. et al. Cell 172, 147-161.e12 (2018) 11. Leibundgut-Landmann, S., Osorio, F., Brown, G. D. \& Reis e Sousa, C. Blood 112, 4971-4980 (2008). 12. Kaufmann, E. et al. Cell 172, 176-190.e19 (2018). 13. Saz-Leal, P. et al. Cell Rep. 25, 1118-1126 (2018). 14. Braza, M. S. et al. Immunity 49, 819-828.e6 (2018). 15. Dijkman, K. et al. Front. Immunol. 10, 2479 (2019). 16. Howard, N. C. et al. Nat. Microbiol. 3, 1099-1108 (2018) 17. Cirauqui, C. et al. Eur. J. Immunol. 48, 180-193 (2018). 18. Quinn, S. M. et al. Front. Immunol. 10, 1109 (2019). 19. Bomans, K. et al. Front. Immunol. 9, 2685 (2018).

Competing interests

The authors declare no competing interests. 